\title{
baroque spiders
}

(on listening to Handel's Tamerlano)

clavinet a metallic spider

miniature lunar lander

binding together the recitatives

on the edge of notice

unremarked

stitching together the threads of the violins

the cellos

the interregnum between arias

—flies trying to break through its web 